\title{
Resilience-based Risk Mitigation for Road Networks
}

\author{
Weili Zhang ${ }^{1}$ and Naiyu Wang ${ }^{2}$
}

\begin{abstract}
:
Transportation infrastructure has been identified by the US Department of Homeland Security as one of sixteen critical infrastructure systems essential to the well-being of modern societies. In this study, we propose a resilience-based framework for mitigating risk to surface road transportation networks. We utilize recent developments in modern network theory to introduce a novel metric based on system reliability and network connectivity to measure resilience-based performance of a road transportation network. The formulation of this resilience-based performance metric (referred in the paper as WIPW), systematically integrates the network topology, redundancy level, traffic patterns, structural reliability of network components (i.e. roads and bridge) and functionality of the network during community's postdisaster recovery, and permits risk mitigation alternatives for improving transportation network resilience to be compared on a common basis. Using the WIPW as a network performance metric, we propose a project ranking mechanism for identifying and prioritizing transportation network retrofit projects that are critical for effective pre-disaster risk mitigation and resilience planning. We further present a decision methodology to select optimal solutions among possible alternatives of new construction, which offer opportunities to improve the resilience of the network by altering its existing topology. Finally, we conclude with an illustration that uses the WIPW as the performance metric to support risk-based mitigation decisions using a hypothetical bridge network susceptible to seismic hazards.
\end{abstract}

Keywords: Bridges; Civil infrastructure systems; Decision optimization; Resilience; Risk mitigation; System reliability; Transportation networks.

\section{Introduction}

The resilience of robust, large-scale, interdependent civil infrastructure networks, including transportation systems, utilities, telecommunication facilities, and social networks, individually and collectively play a major role in determining the resilience of a community as a whole. The performance of transportation networks, in particular, is critical because post-disaster restoration of virtually all other facilities and lifelines in a community depends on people and equipment being able to move to the sites where damage has occurred. Highway bridges typically are the vulnerable links in road transportation systems and require

\footnotetext{
${ }^{1}$ Graduate Research Assistant, Department of Industrial and Systems Engineering, University of Oklahoma, USA weili.zhang-1@ou.edu

${ }^{2}$ Assistant Professor, School of Civil Engineering and Environmental Science, University of Oklahoma, USA naiyu.wang@ou.edu; Corresponding Author
} 
especially effective risk mitigation strategies aimed at improving the overall resilience of transportation systems against future natural disasters.

The resilience of a system is its ability to withstand or adapt to external shocks and to recover from such shocks efficiently and effectively (Timmerman, 1981; Pimm, 1984). In the case of civil infrastructure, resilience is often associated with four attributes (Bruneau et al 2003; Chang and Shinozuka, 2004): robustness - the ability to withstand an extreme event and deliver a certain level of service even after the occurrence of that event; rapidity - to recover the desired functionality as quickly as possible; redundancy - the extent to which elements and components of a system can be substituted for one another; and resourcefulness - the capacity to identify problems, establish priorities, and mobilize personnel and financial resources after an extreme event. These attributes are illustrated in Figure 1a; all are characterized by considerable uncertainties. Many research studies have discussed the resilience of systems other than civil infrastructure, including ecosystems (Holling, 1973; Walker and Salt, 2006; Leichenko, 2011), computer networks (Scheffel, et al 2006; Simonis, 2006), communication networks (Sterbenz et al 2010; Zhu and Basar, 2015), and socio-economic systems (Ross, 2007; Martin, 2012).

Strategic investments and mitigation strategies can gradually improve the resilience of a system against future disasters, as indicated in Figure 1b. For road transportation systems, such risk mitigation strategies often involve rehabilitation or retrofit of network bridges. However, the engineering processes of retrofitting can be very costly and time consuming, and retrofit decisions are often constrained by limited financial and human resources. Consequently, systematic retrofit prioritization is a critical element for an effective risk mitigation framework. Such a framework requires not only a consideration of the physical condition and structural vulnerability of each individual bridge in the network (e.g. Shinozuka et al, 2000; Padgett and DesRoches, 2009) but also a system perspective that takes into account the overall pre- and post- disaster operation and functionality of the network as a whole (e.g. Sohn et al, 2003; Nagurney and Qiang, 2007; Peeta et al, 2010). As examples, Shiraki et al (2007) combined bridge fragility curves with network user-equilibrium functions to estimate the total road network delay due to earthquake-introduced damages; Bocchini and Frangopol (2013a) proposed a bridge network maintenance scheduling approach that incorporated both individual bridge reliabilities and the network connectivity into a decision optimization formulation. Ghosh et al. (2014) presented a two-stage reliability assessment framework for aging bridge networks, including seismic fragilities of individual bridges and correlations among them, and further estimated the network reliability by a revised Markov Chain Monte Carlo simulation. The proposed method is illustrated on part of the highway bridge network in South California, USA (Rokneddin et al., 2014). 


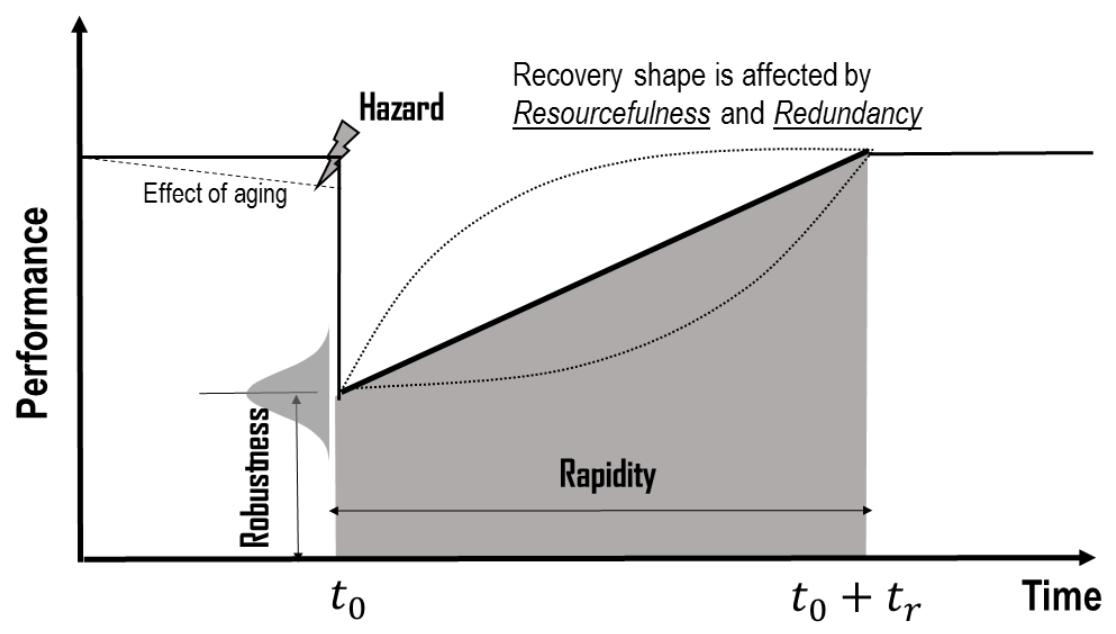

(a)

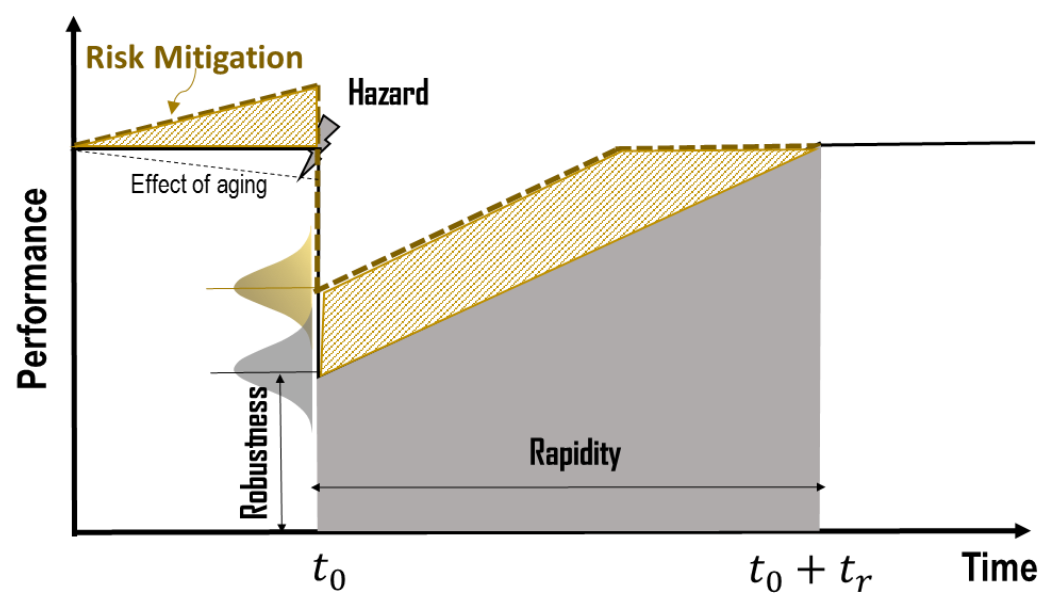

(b)

Figure 1. Illustration of (a) the concept of resilience and (b) the effect of risk mitigation plan

As illustrated in Figure 1, any resilience-based analysis and decision require a quantitative measure of the system performance and condition (i.e. the vertical coordinates in the Figure 1). While structural reliability (failure probability) is a well-accepted performance measure for individual roads and bridges to natural hazards, the performance of a transportation network must be measured by different metrics. Many researchers have quantified network performance based on network service functions, e.g., flow capacity (Nagurney and Qiang, 2007; Lee et al., 2011), connectivity (Chen et al. 2002; Clark and Watling, 2005; Guikema and Gardoni, 2009; Bocchini and Frangopol, 2013a; Kurtz et al., 2015), and travel time (Asakura and Kashiwadani, 1995; Chen et al., 2007; Zhang and Wang, 2015). However, these metrics are mainly used to measure network performance under normal service conditions and are not effective in reflecting 
the network susceptibility to disruptive, low-probability high-consequence natural and man-made hazards or its resilience (earthquakes, floods, terrorist attacks, etc.). More recently, Peeta et al (2010) used postdisaster connectivity and traversal cost between multiple origin-destination pairs in a network as the basis for pre-disaster investment decisions. Morlok and Chang (2004) proposed capacity flexibility to reflect a transportation system's ability to adapt to changes in traffic patterns caused by natural disasters. Chang and Nojima (2001) introduced the notion of network coverage and transport accessibility as the performance measures for post-disaster network recovery. Ip and Wang (2011) suggested that pathway redundancy between all origin-destination pairs be used as a resilience measure for transportation networks. These performance metrics all have their merits in quantifying the network performance under hazardous conditions. However, none of them individually can reflect the network resilience-based performance in terms of its ability to provide functionality to community following a disaster and to support community recovery decisions from hazard-induced interruptions. Furthermore, none of these studies has attempted to quantify the uncertainties associated with these performance metrics. Different metrics might be appropriate for different decisions (e.g. retrofit, repair, new construction, etc.) at different stages (e.g., preevent, immediately following event, and long-term recovery ) of network resilience planning. Uncertainties must be quantified to ensure these decisions are risk-informed.

\section{Organization and Highlights of the Paper}

In this paper, we propose a novel resilience-based performance metric for road transportation networks, which allows resilience-based risk mitigation alternatives to be measured and compared on a common basis. The performance metric is based on graph theory, in a formulation which systematically integrates the network topology, system redundancy, traffic patterns, reliability (failure probability) of network components (i.e. bridges and roads) and the network functionality in a community's immediate post-disaster recovery period. Based on this resilience-based performance metric, we next introduce a project ranking mechanism for identifying and prioritizing bridge retrofit projects that are critical for effective pre-disaster risk mitigation of road transportation networks. We provide a decision methodology to select optimal solutions among possible alternatives of new construction which offer opportunities to improve network resilience by altering its existing topology. We conclude with an illustration of a risk-based mitigation framework, considering a hypothetical networked system of 37 bridges that are susceptible to seismic hazard.

\section{Resilience-based Network Performance Metric, WIPW}

The fundamental purpose of a transportation system is to carry traffic from origins to destinations. The resilience of such a system is reflected in its ability to continue to fulfill this purpose in the event of natural 
or man-made disasters. Extreme hazard events can damage many bridges and roads simultaneously in a local transportation network, and financial and human resources required to restore the network function often are not immediately available following the disaster. Thus, the existence of redundant alternative paths between network origin-destination (O-D) pairs is crucial for the continued function of the transportation system during the period of emergency response immediately following the disaster as well as the long-term recovery of the community, and is an essential characteristic of a resilient transportation network.

Accordingly, by extending the concept suggested by Ip and Wang (2011), we define a resiliencebased performance metric of a transportation system as the weighted average number of reliable independent pathways between any network O-D pairs. A pathway between an O-D pair usually consists of several links that represent roads, with or without a bridge, which are connected in series. Two pathways between the same O-D pair are considered as independent pathways (IPW) if they do not share any common road links. Although the number of pathways between an O-D pair can be very large, the number of IPWs is often very limited. The process of identifying all IPWs in a transportation network will be discussed later. We select IPW rather than all-pathways (APWs) as the base for the subsequently introduced new resilience-based performance metric because: (i) for a given network, the number of IPW is limited (Ip and Wang, 2011), but the number of APWs is likely to be very large. When a large number of pathways all share common links, the number of APWs between an O-D pair can be overly sensitive to the damage to those commonly shared links, therefore is not a stable metric of the overall network performance; and (ii) finding APWs between two nodes is NP-Hard, and therefore is impractical for decision making practice. Note that IPWs for different O-D pairs or even between a same O-D pair may not have identical impacts on the network performance. The resilience-based performance metric, as formulated subsequently and referred as WIPW, includes a novel and systematic weighting mechanism to quantitatively reflect the contribution of each IPW to the overall network performance under hazard consideration.

Introducing the terminology of graph theory (Gibbons, 1985), let $G=(V, A)$ denote the road network, where $V=\{1,2 \ldots n\}$ is the set of nodes that represents major road intersections and economic hubs and key destinations in a community, and $A=\{1,2 \ldots m\}$ is the set of arcs (links) that represent roads either without a bridge or with a maximum of one bridge ${ }^{3}$. The network performance metric, WIPW, as defined above, can be written as:

\footnotetext{
${ }^{3}$ In WIPW formulation a link is a segment that can only have one bridge at most, because the mitigation decision is made on individual bridges (links), each of which need a unique identity in the network topology representation.
} 


$$
W I P W(G)=\sum_{i=1}^{n} w_{i} r_{i}
$$

where $w_{i}=$ weighting factor applied to individual node $i \in V, \sum_{i=1}^{n} w_{i}=1$, and $r_{i}=$ the average number of reliable IPWs between node $i$ and any other $n-1$ nodes in the network, as expressed in Eq. (2):

$$
r_{i}=\frac{1}{n-1} \sum_{j=1, j \neq i}^{n} \sum_{k=1}^{K_{(i, j)}} w_{k}(i, j) \cdot R_{k}(i, j)
$$

in which $K_{(i, j)}=$ total number of IPWs between nodes $i$ and $j ; R_{k}(i, j)=$ reliability of $\operatorname{IPW} P_{k}(i, j)$, the $k$ th IPW between node $i$ and node $j ; w_{k}(i, j)=$ weighting factor applied to IPW $P_{k}(i, j)$, and for all $K_{(i, j)}$ IPWs between nodes $i$ and $j, \sum_{k=1}^{K_{(i, j)}} w_{k}(i, j)=K_{(i, j)}$. Weighting factors $w_{i}$ and $w_{k}(i, j)$ will be discussed in detail below. Each IPW, $P_{k}(i, j)$, usually consists of several road links connected in series. Let $l$ denote the individual road links and $q_{l}$ denote the reliability of $l$; thus, for a series that consists of independent links, the system reliability of the series is the product of the reliabilities of all road links included in $P_{k}(i, j)$ :

$$
R_{k}(i, j)=\prod_{\forall l \in P_{k}(i, j)} q_{l}
$$

For a system in which the component performances are positively correlated, which is likely the case for transportation networks (further discussion is provided in Section 4), Eq. (3) provides a lower bound on system reliability. The exact system reliability $R_{k}(i, j)$ can be estimated through Monte Carlo Simulation using a Gaussian Copula to model correlation. Combining Eqs (1) and (2), the resilience-based performance metric of the road network, WIPW, becomes

$$
W I P W(G)=\sum_{i=1}^{n} w_{i} \frac{1}{n-1} \sum_{j=1, j \neq i}^{n} \sum_{k=1}^{K_{(i, j)}} w_{k}(i, j) \cdot R_{k}(i, j)
$$

Two weighting factors, $w_{i}$ and $w_{k}(i, j)$, appear in Eq. (4). Factor $w_{i}$ applies to nodes; it is inversely proportional to the shortest distance from node $i$ to the nearest emergency response facility in the community, reflecting the relative importance of the node $i$ being connected in the context of community post-disaster emergency response. Let $E$, a subset of $V$, denote the set of nodes in the network where emergency response facilities are located, $N=$ the set of nodes that do not belong $E$, and $L_{\mathrm{P}_{k}(i, j \in E)}=$ the length of $P_{k}(i, j)$ where $j \in E$. We then evaluate $w_{i}$ as: 


$$
w_{i}=\frac{\Omega_{i}}{\sum_{j=1}^{n} \Omega_{j}}
$$

where

$$
\Omega_{i}= \begin{cases}\frac{1}{\min \left\{L_{P_{k}(i, j \in E)} \mid k=1,2 \ldots K_{(i, j \in E)}\right\}}, & i \in N \\ 1, & i \in E\end{cases}
$$

As noted previously, the sum of $w_{i}$ for all the nodes in the network equals 1 .

The other weighing factor $w_{k}(i, j)$ in Eq. (4) applies to IPWs; it is related to both the average daily traffic (ADT) and the length of the IPW, and reflects the relative impact that this pathway has on people's normal life activities and the local economy. Pathways between any given O-D pair that has shorter length and carries larger traffic flow contribute more to the network functionality and should be weighted more heavily in quantifying the network resilience. ADT data is often readily available with federal, state or local bridges owners, or can be estimated using traffic assignment models. Let $T_{l}$ denote the ADT of road link $l \in \mathrm{P}_{k}(i, j)$. Define $T_{\mathrm{P}_{k}(i, j)}$, the ADT of IPW $\mathrm{P}_{k}(i, j)$, as the minimum ADT of all road links on that pathway:

$$
T_{\mathrm{P}_{k}(i, j)}=\min \left[T_{l} \mid l \in \mathrm{P}_{k}(i, j)\right]
$$

The normalized ADT of the path is then defined as:

$$
T_{\mathrm{P}_{k}(i, j)}^{\prime}=\frac{T_{\mathrm{P}_{k}(i, j)}}{\sum_{k=1}^{K(i, j)} T_{\mathrm{P}_{k}(i, j)}} \times K_{(i, j)}
$$

Note that for any node pair $(i, j), \sum_{k=1}^{K_{(i, j)}} T_{\mathrm{P}_{k}(i, j)}^{\prime}=K_{(i, j)}$. Similarly, let $L_{l}=$ the length of the road link $l$; then the length of the IPW $P_{k}(i, j)$ is simply the summation of the lengths of all road links within that path:

$$
L_{\mathrm{P}_{k}(i, j)}=\sum_{l \in \mathrm{P}_{k}(i, j)} L_{l}
$$

Finally, let $L_{\max }(i, j)=$ maximum of all $L_{P_{k}(i, j)}$ for a given O-D pair $(i, j)$; then the normalized length of the path is:

$$
L_{\mathrm{P}_{k}(i, j)}^{\prime}=\frac{L_{\max }(i, j)}{L_{\mathrm{P}_{k}(i, j)} \cdot \sum_{k=1}^{K(i, j)}\left(L_{\max }(i, j) / L_{\mathrm{P}_{k}(i, j)}\right)} \times K_{(i, j)}
$$


Note that for any node pair $(i, j), \sum_{k=1}^{K_{(i, j)}} L_{\mathrm{P}_{k}(i, j)}^{\prime}=K_{(i, j)}$. Using Eqs (8) and (10), we define the aggregated pathway weighting factor $w_{k}(i, j)$ as:

$$
\left.w_{k}(i, j)=u \cdot L_{\mathrm{P}_{k}(i, j)}^{\prime}+(1-u) \cdot T_{\mathrm{P}_{k}(i, j)}^{\prime}\right)
$$

where $u \in[0,1]$ is a weighting factor to impose the relative importance between the pathway length and its ADT. A community (or government decision makers) can assign different values to $u$ based on their preferences in order to obtain the "best" measure to their specific situation. For the illustration presented in the subsequent section, we simply apply equal weights, i.e., $u=0.5$. Note that the summation of all $w_{k}(i, j)$ for a given O-D pair $(i, j)$ equals $K_{(i, j)}$, the total number of IPWs between nodes $i$ and $j$.

The node weighing factor $w_{i}$ and IPW weighting factor $w_{k}(i, j)$, so defined, not only ensure that all nodes and links in the network are properly weighted in the resilience-based performance metric based on their individual attributes (topology, traffic patterns, and functionality during a community's postdisaster recovery as well as structural reliability of individual bridges), but also preserve the physical meaning of the metric WIPW - the weighted average number of reliable IPWs between all O-D pairs in the road network. The role that a transportation system plays before, during and after a disaster varies uniquely for every community. Communities of different size, population and social-economic attitudes and vulnerabilities are likely to show different values and preferences when evaluating the performance of their transportation systems. The weighting mechanism, formulated as above, provide a transparent framework to incorporate and properly weigh other network attributes in addition to those discussed herein that might be valuable to a specific community.

Figure 2 displays the algorithm for computing WIPW. The identification of IPWs between many O-D pairs is non-unique, depending on the algorithm or process used to search for IPWs. To mitigate this problem, we apply Dijkstra's algorithm (Skiena, 1990), as highlighted in Figure 2 with a dashed-line box, to search for a succession of shortest independent paths. The justification for the shortest IPW is that people usually has the preference to choose the shortest path when traveling, but the WIPW, in and of itself, is not necessarily bonded to the Dijkstra's algorithm. 


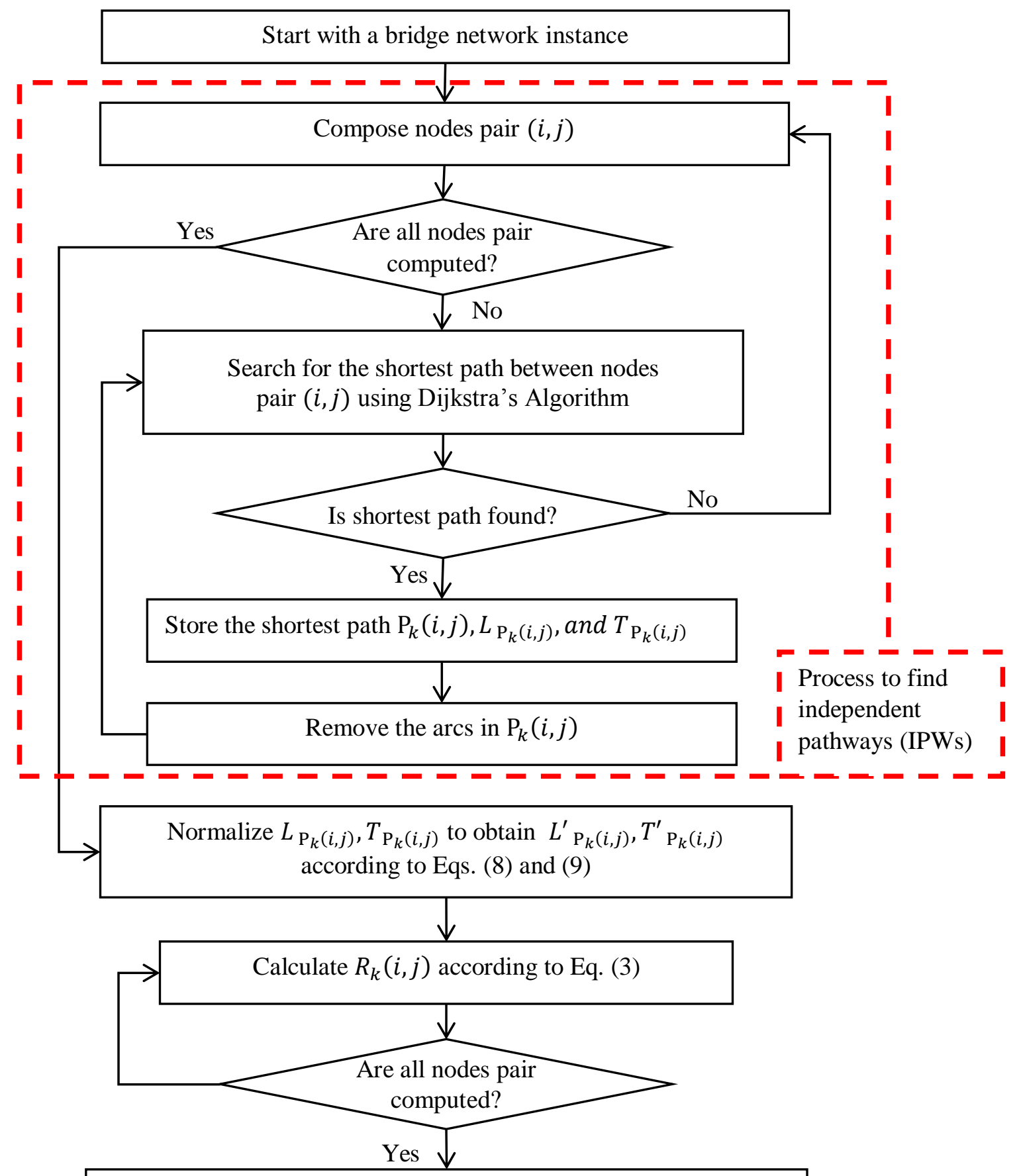

Compute $r_{i}$ according to Eq. (2), and $\Omega_{i}$ and $w_{i}$ to Eqs. (6) and (7)

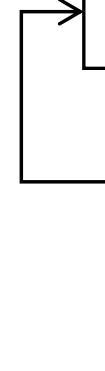




\section{Reliability of Bridges (Links) in a Community Transportation System}

An important step to quantify the WIPW is to properly assign link (i.e. road segments with or without a bridge) reliabilities. The reliability of an individual bridge or road segment can be evaluated using fragility analyses associated with damage states of interest [e.g. using platform HAZUS-MH MR4 (DHS 2009)]. The calculated WIPW is therefore hazard-specific because the link reliabilities are hazard specific. It should be recognized that hazardous events with large footprints introduce spatial and temporal correlations to the demands on community infrastructure systems (Adachi and Ellingwood, 2008; Jayaram and Baker, 2009; Ghosh et al., 2014). Common construction practices and code enforcement within a community also introduce positive correlation in structural response above and beyond that introduced by the hazard (Vitoontus and Ellingwood, 2013; Bonstrom and Corotis 2014). Such correlation structures depend on the stochastic variability in the hazard demand, the locations of bridges and roads, and their susceptibility to damage if the hazardous event occurs, and need to be taken into consideration in the probabilistic evaluation of the network performance (Lee and Kiremidjian, 2007; Bocchini and Frangopol, 2011).

Bridge fragility assessment is an important ingredient for seismic risk assessment of transportation infrastructure (Padgett and DesRoches, 2009). The current research is focused on transportation network performance assessment. Because such networks may contain many different types of bridges, bridge fragility modeling is outside the scope of the current effort. However, a practical assessment of the integrity of any transportation network should contain specific fragility models for the key bridges in the network. In the subsequent examples, we use plausible estimates of failure probabilities for generic bridge types.

\section{Formulation of Optimal Risk Mitigation Strategies}

The quantitative resilience-based performance metric, WIPW, as formulated in Section 3, can be employed to evaluate the transportation network performance, and it can also be incorporated in a decision framework to provide a common basis for evaluating and comparing alternative risk mitigation strategies on a common and rational basis. Possible pre-disaster risk mitigation strategies to improve road network resilience include retrofitting existing bridges (links) or building new bridges. In either case, the formulation of the decision process is the same: to make selections from a set of candidate links, representing either existing bridges and road segments or potential new construction, to maximize the network performance WIPW and, simultaneously, to minimize the associated cost.

Suppose the set of candidates is represented by $S=\left\{s_{1}, s_{2} \ldots s_{S}\right\}$ and the corresponding cost of each is $c=\left\{c_{1}, c_{2} \ldots c_{s}\right\}$. Let $x_{t} \in X$, where $t=1,2 \ldots s$, denote the decision variables as below, 


$$
x_{t}=\left\{\begin{array}{rr}
1, & s_{t} \in S \text { is selected } \\
0, & \text { otherwise }
\end{array}\right.
$$

Since the risk mitigation strategy (or decision) represented by $X$ will upgrade the existing network, we rewrite all the parameters of the upgraded network in the form of argument $X$, e.g. , $W I P W(G(X)), w_{i}(X), r_{i}(X)$ and $\mathrm{P}_{k}(i, j \mid X)$. Furthermore, some network parameters are uncertain and can be treated as random variables in the problem formulation; these variables will be represented with argument $\xi$. Thus, the first objective of the decision process is to maximize the network performance metric WIPW:

$$
\max W \operatorname{IP} W(G(X, \xi))=\sum_{i=1}^{n} w_{i}(X) r_{i}(X, \xi)
$$

Let $\theta(X)$ denote the cost associated with decision $X$; the second objective function is to minimize the total cost:

$$
\min \theta(X, \xi)=\sum_{t=1}^{s} c_{t}(\xi) x_{t}
$$

Eqs. (13) and (14) pose a nontrivial multi-objective optimization problem, and a single solution that simultaneously optimizes these two competing objectives, i.e., maximizes WIPW and minimizes cost associated with mitigation strategies, does not exist. However, a (possibly infinite) number of Paretooptimal solutions do exist, which allows the tradeoff between the competing objectives and the subjective preferences of a decision maker to be factored into the decision process. To compute the objective function of Eq. (13) requires the algorithm described in Figure 2, a non-closed form formulation, which requires metaheuristic techniques to search for near-optimal solutions. Accordingly, we use a Non-dominated Sorting Genetic Algorithm-II (NSGA-II) (Deb et al, 2002) to search for the Pareto frontier; NSGA-II have been successfully applied to search for near-optimal solutions of similar network problems (Pishvaee, et al., 2010; Mesbah et al, 2011, Osman and Kelly, 2012). We couple the NSGA-II with Monte Carlo Simulation (MCS) to account for uncertainties in the optimization process in the subsequent case study. Since the parameter values have significant effects on the performance of the NSGA-II (Eiben and Smit, 2012), we performed preliminary tests ${ }^{4}$ on parameter tuning, resulting in the mutation parameter, crossover rate and population size in the NSGA-II to be set to $0.1,0.7$ and 100, respectively. The maximum number of iterations is 1000 , and the early termination criterion is 50 , which means the program will stop if no better

\footnotetext{
${ }^{4}$ Different combinations of parameters of NSGA-II are examined with 100 iterations and the best one is selected regarding the solution quality and speed of convergence.
} 
solution is found in consecutive 50 iterations. To improve the performance of the NSGA-II, the initial generation is diversified with respect to the percentage of selected bridges among all candidates.

\section{Illustration - Risk-based Mitigation Decisions of Transportation Network Exposed to Seismic Hazards}

In this section, the role of the network resilience-based performance metric and the application of the decision methodology for risk mitigation are illustrated with a hypothetical road network exposed to a severe earthquake. Two scenarios are discussed: (1) establishing priorities for pre-disaster retrofitting bridges that are critical for transportation network resilience; and (2) selecting among possible alternatives of new constructions which offer opportunities to improve the resilience of the network by altering its existing topology.

\subsection{Road/Bridge Network}

Figure 3 illustrates a hypothetical community road system, with 37 links representing the roads and 30 nodes representing the major road intersections and economic hubs. The community emergency response facilities (e.g. fire stations, hospitals, police, etc.) are located at Nodes 9 and 17. For simplicity, we further assume that every road contains exactly one bridge. This assumption can be easily relaxed, if necessary. If all the bridges in the network are in "as new" condition and reliabilities under service loads are assumed equal to 0.999 , we find the mean pre-event WIPW is 1.70 , which means that on average there are 1.70 reliable IPW between any O-D pair in the road network under normal operational conditions.

We now assume that a severe earthquake occurs, with magnitude $\mathrm{M}_{\mathrm{W}}$ equal to 7 and epicentral distance approximately $40 \mathrm{~km}$ from the centroid of the network (close to Node 13). For illustrative purposes, we assume that out of the 37 bridges, 19 are steel (S) bridges and 18 are reinforced concrete (RC) bridges. To reflect the diversity of bridge construction (including bridge configuration, material and area) within the network, we assume that the mean reliabilities of the 19 steel bridges and $18 \mathrm{RC}$ bridges under the considered seismic action are 0.80 and 0.70 , respectively. Furthermore, to reflect the epistemic uncertainty associated with the estimate of bridge reliability, the reliability for each bridge is described by a normal distribution, with a mean value as tabulated in Table 1 and a coefficient of variation of 0.07 for all bridges; the implication of this assumption, often made in probabilistic risk analysis, is that the reliability of each bridge in this system can be estimated to within $\pm 15 \%$ with more than $95 \%$ confidence. This modeling approach results in a bridge portfolio that represents a diversity of construction practices. Correlations exist in the demand and capacity of these bridges as discussed in Section 4 due to common hazard and similar construction code and practices. These correlations are modeled as exponentially decreasing with the 
increase in the separation distance between bridges, i.e. $\rho_{i j}=\exp \left(-a_{i j} / L_{c}\right)$ (Vitoontus and Ellingwood 2013), where $a_{i j}$ is the separation distance between bridges $i$ and $j$, and $L_{c}$ is the correlation length which is set to be the largest distance between two bridges in the network. This spatial correlation model reflects the fact that correlation in bridge performance due both to seismic demand and common construction practices is likely to diminish as their separation distance increases. In addition, the retrofit cost for each bridge is also modeled with a normal distribution, assuming that the mean cost is a function of the bridge deck area and its structural reliability (Fragkakis and Lambropoulos, 2004), and coefficient of variation is 8\%. The mean reliability, mean retrofit cost and mean ADT of each bridge are tabulated in Table 1 . The distributions used to model the uncertainty associated with these parameters are assumed and summarized in Table 2.

Figure 4 illustrates the histogram of the network WIPW under the considered hazard scenario, obtained from 1,000 Monte Carlo simulations of the network performance, which was found through experimentation to yield a stable estimate of the mean WIPW. The mean residual WIPW of the current network without any risk mitigation actions is 0.61 , and its coefficient of variation (COV) is 0.22 . That is, if the scenario earthquake were to occur, the average number of weighted independent pathways between all O-D pairs is less than 1, meaning that some areas in the community will become isolated from one another. This analysis provides a baseline for comparing the effectiveness of the alternative risk mitigation strategies to be evaluated in the subsequent section. 


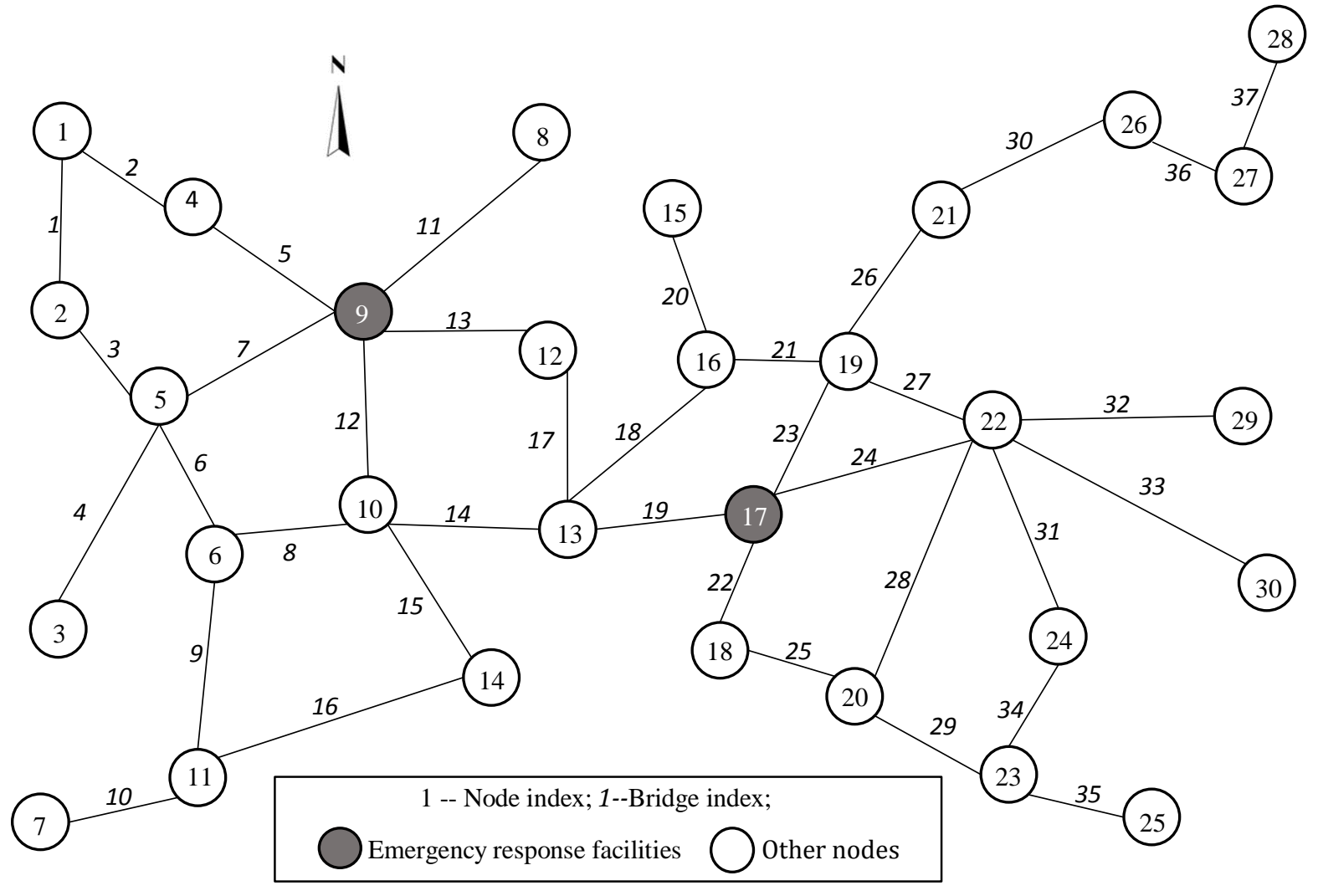

Figure 3. Hypothetical Bridge Network

Table 1. Mean Values of Network Parameters

\begin{tabular}{ccccc}
\hline Bridge ID & $\begin{array}{c}\text { Construction } \\
\text { Type }\end{array}$ & Reliability & $\begin{array}{c}\text { ADT } \\
\text { (Vehicle/Hour) }\end{array}$ & Cost (Unit) \\
\hline 1 & RC & 0.66 & 2200 & 3.57 \\
2 & RC & 0.76 & 1900 & 3.82 \\
3 & S & 0.82 & 2000 & 4.34 \\
4 & S & 0.88 & 1500 & 4.17 \\
5 & RC & 0.55 & 1900 & 4.87 \\
6 & S & 0.84 & 2200 & 3.49 \\
7 & S & 0.77 & 700 & 4.41 \\
8 & S & 0.82 & 2400 & 3.74 \\
9 & S & 0.77 & 2600 & 2.61 \\
10 & S & 0.85 & 300 & 3.55 \\
11 & S & 0.84 & 800 & 2.53 \\
12 & RC & 0.71 & 900 & 5.29 \\
13 & S & 0.89 & 2500 & 4.78 \\
14 & S & 0.78 & 600 & 3.25
\end{tabular}




\begin{tabular}{ccccc}
\hline Bridge ID & $\begin{array}{c}\text { Construction } \\
\text { Type }\end{array}$ & Reliability & $\begin{array}{c}\text { ADT } \\
\text { (Vehicle/Hour) }\end{array}$ & Cost (Unit) \\
\hline 15 & RC & 0.77 & 2000 & 3.43 \\
16 & S & 0.78 & 500 & 4.33 \\
17 & RC & 0.61 & 2500 & 3.14 \\
18 & RC & 0.79 & 2800 & 2.98 \\
19 & S & 0.80 & 1300 & 2.88 \\
20 & S & 0.75 & 1700 & 3.28 \\
21 & S & 0.89 & 1500 & 3.98 \\
22 & S & 0.81 & 1200 & 4.82 \\
23 & RC & 0.76 & 1500 & 3.24 \\
24 & S & 0.75 & 700 & 4.80 \\
25 & S & 0.78 & 1800 & 3.80 \\
26 & S & 0.75 & 900 & 4.66 \\
27 & S & 0.80 & 600 & 4.46 \\
28 & RC & 0.71 & 800 & 3.33 \\
29 & RC & 0.65 & 1400 & 4.86 \\
30 & RC & 0.67 & 2800 & 3.45 \\
31 & RC & 0.69 & 1900 & 3.08 \\
32 & RC & 0.75 & 2900 & 3.74 \\
33 & RC & 0.79 & 1300 & 4.50 \\
34 & RC & 0.69 & 900 & 4.47 \\
35 & RC & 0.72 & 2200 & 3.36 \\
36 & RC & 0.83 & 700 & 4.46 \\
37 & RC & 0.73 & 3000 & 5.15 \\
\hline & & & & \\
\hline
\end{tabular}

Table 2. Statistics of the Network Parameters

\begin{tabular}{lclcc}
\hline Parameters & Notation & Distribution & Mean & COV \\
\hline Individual Bridge Reliability & $q$ & Normal & & 0.07 \\
Average Daily Traffic (ADT) & $T$ & Uniform & As tabulated In & 0.05 \\
Retrofit Cost & $c$ & Normal & Table 1 & 0.08 \\
\hline
\end{tabular}




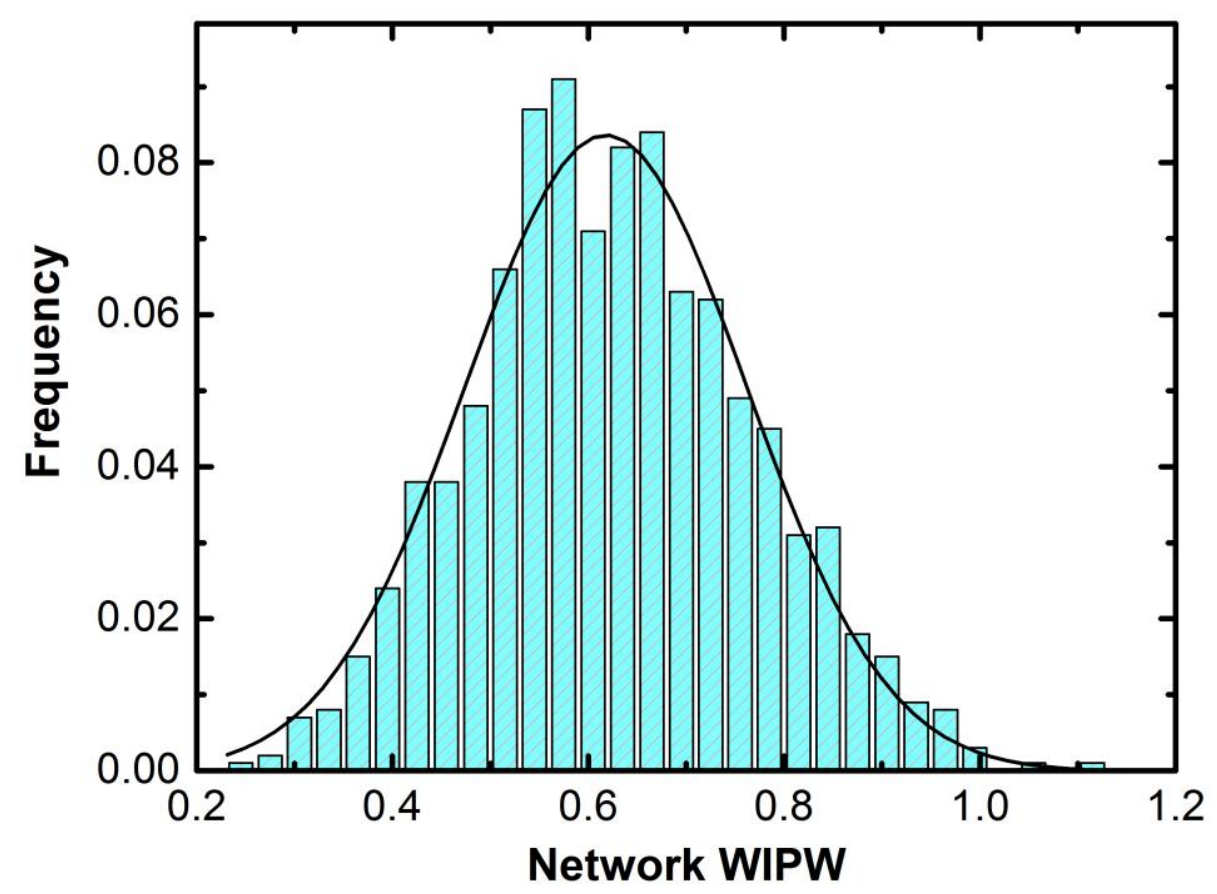

Figure 4. Histogram of Network WIPW before Retrofit

\subsection{Improving network resilience by strengthening critical bridges}

When only limited resources are available for risk mitigation aimed at achieving a more resilient network through bridge retrofitting, it is critical to distribute those resources within the network in such a way that the overall network performance is maximized. Since there are 37 bridges in the network and each can be either selected or not selected for retrofit, the solution space has $2^{37}$ different combinations. As discussed previously, the GA is used to solve this multi-objective optimization problem as formulated in Section 5. We assume that a retrofit invention will bring the reliability a bridge back to 0.999 , the "as new" condition.

Figure 5 shows the tradeoff between network performance and financial investment in risk mitigation, with the triangular markers representing the collection of optimal solutions (Pareto Frontier) when WIPW and retrofitting cost (measured in non-dimensional cost units) are considered as competing objectives. With a total of 150 cost units, the alternative shown at the top right corner of the Figure 5 selects all 37 bridges for retrofit and the corresponding network WIPW increases to the pre-earthquake value of 1.7, as all bridges are upgraded to the "near-perfect" hazard-resilient condition. Four solutions on the Pareto Frontier, marked as I, II, III and IV in Figure 5, are identified in Table 3 for further examination. Generally speaking, a larger financial investment will lead to more bridges being selected for retrofit and, 
consequently, a more resilient network. For instance, Solution I identifies seven bridges in the network as having retrofit priority, with a budget of 25 cost units, resulting a network WIPW of 0.76 . In contrast, Solution III indicates that with a budget of 86 cost units, 22 bridges can be selected for retrofit, leading to a WIPW of 1.23 , which is a $101 \%$ increase over the pre-retrofit WIPW of 0.61 . Figure 6 compares the network WIPW before and after the retrofit associated with Solution III when uncertainties are considered in the evaluation.

Table 3 reveals that the increase in the number of selected bridges from Solution I to Solution IV is not simply due to adding more bridges to the selected group associated with a lower budget. The likelihood that some bridges, e.g. bridges 11 and 13, are selected increases as the budget increases. Other bridges, e.g. bridges 4 and 33, are selected initially when the budget is low; however, they are de-selected as the budget increases because a neighboring bridge on an alternative path may become a more costeffective candidate for improving the overall network performance. Finally, bridges 5 and 17 are always selected in optimal retrofit solutions because, when compared with other bridges, they: (1) have much lower than average structural reliabilities (0.55 and 0.61); (2) carry heavy ADT (1900 and 2500); (3) are shared by IPWs between multiple node-pairs; and (4) are in close proximity to emergency response facilities. This budget-specific prioritization mechanism holistically integrates the network characteristics and individual bridge properties in resilience-based decision for the road network. 


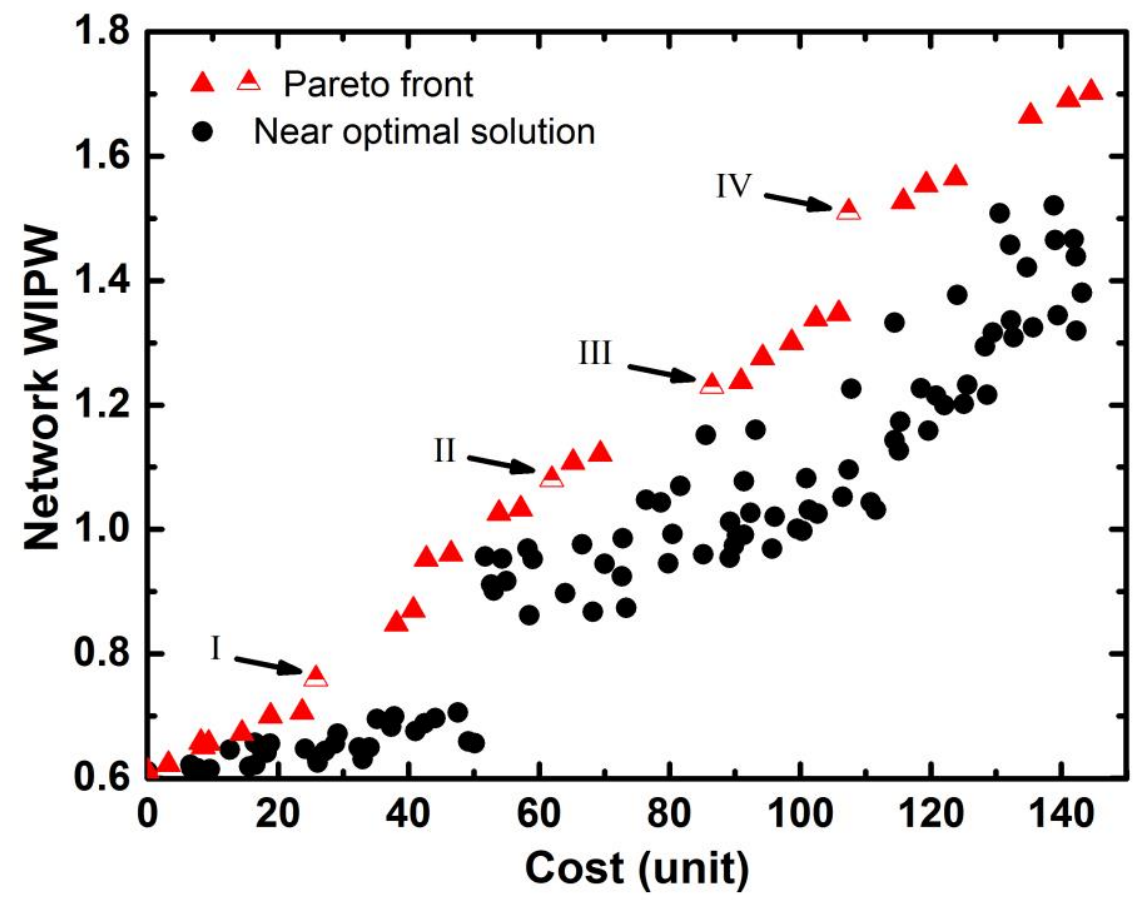

Figure 5. Pareto Frontier of Optimal Solutions

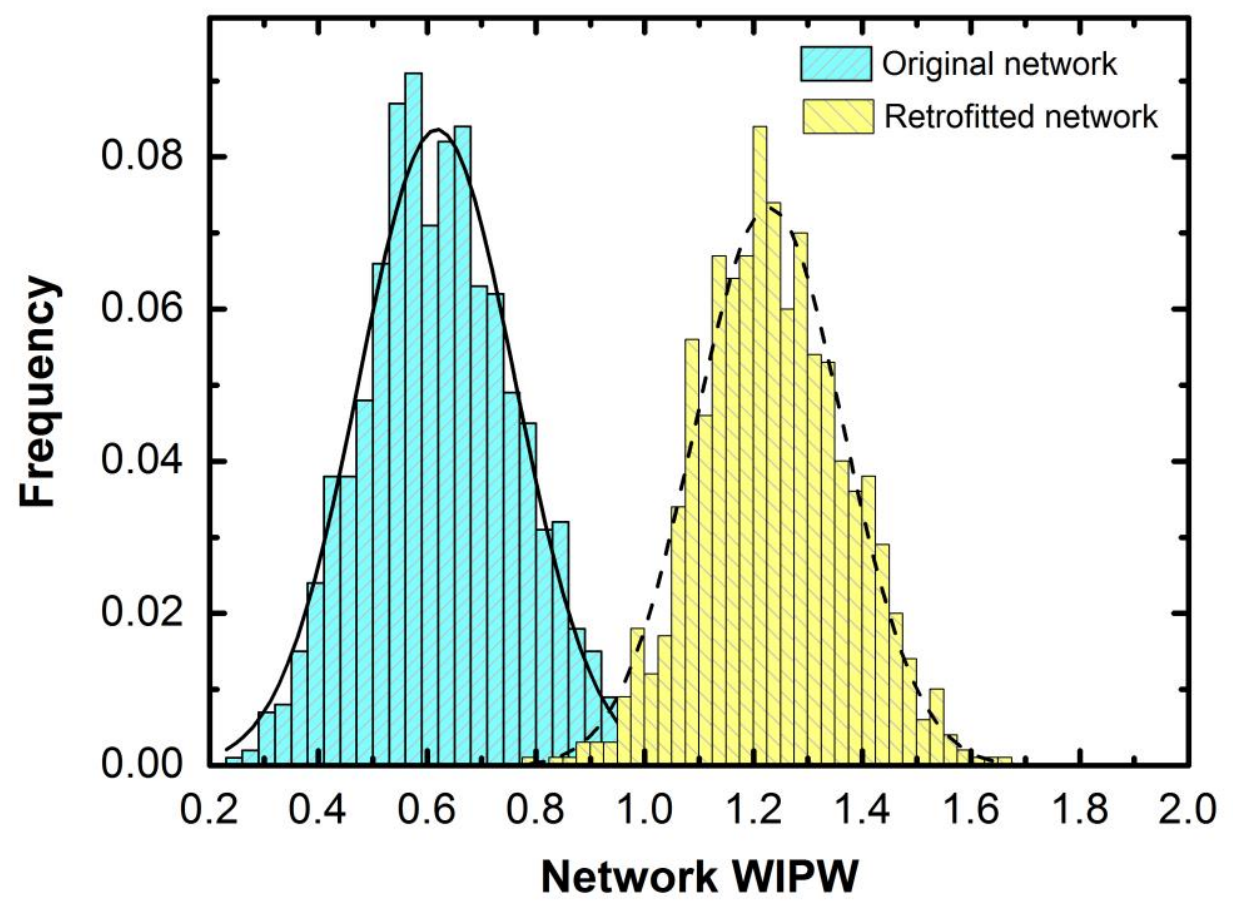

Figure 6. Comparison of Network WIPW before and after Retrofit (Solution III) 
Table 3. Details of Four Optimal Solutions in Pareto Frontier

\begin{tabular}{|c|c|c|c|c|}
\hline $\begin{array}{l}\text { Solution } \\
\text { ID }\end{array}$ & Cost & $\begin{array}{l}\text { Mean } \\
\text { WIPW }\end{array}$ & $\begin{array}{l}\text { Number of bridges } \\
\text { selected for retrofit }\end{array}$ & Bridges Selected \\
\hline I & 25 & 0.76 & 7 & $5,17,8,32,10,4,33$ \\
\hline II & 62 & 1.07 & 14 & $\begin{array}{l}22,5,28,17,20,14,12,19,27,15 \\
24,16,36,21,37,31\end{array}$ \\
\hline III & 86 & 1.24 & 22 & $\begin{array}{l}27,11,19,16,10,31,5,17,18,37, \\
23,30,28,14,12,24,21,22,29,36, \\
15,20\end{array}$ \\
\hline IV & 107 & 1.51 & 27 & $\begin{array}{l}7,6,11,19,27,31,1,5,17,8,18 \\
37,25,26,23,30,28,14,2,24,21 \\
35,22,29,36,20,13\end{array}$ \\
\hline
\end{tabular}

\subsection{Improving network resilience by changing network topology through new construction}

Opportunities may exist in some communities, especially in developing counties, to improve the resilience of existing transportation networks through new construction. For example, the hypothetical road network considered herein has two obvious areas of potential weakness as far as resilience is concerned. The first weakness is the branch from node 19 to node 28, highlighted with thickened lines in Figure 7, where four bridges - 26, 30, 36, and 37- are connected in series to the rest of the network through node 19. If any of the four bridges fail due to the earthquake, the area to the east of that bridge will be isolated from the rest of the community because an alternative path does not exist. The second weakness is if both bridges 18 and 19 fail, the entire community road system will be divided into two unconnected subsystems, and the resilience of the road system will instantly drop to 0.39 . Relieving the burden on these two bridges by creating bypasses will increase the overall resilience of the network.

Suppose that as part of community development, links S1-S5, shown in dashed lines on Figure 7, are proposed as candidates for new construction to mitigate the above-mentioned potential risks. Suppose, further, that resources are available to construct only two additional roads out of the five candidates: one road to be selected from S1, S2, or S3 to increase the connectivity of the branch from node 19 to node 28 , and the other to be selected from S4 or S5 to relief the burden on roads 18 and 19. The decision method outlined in Section 5 is applied to select from the alternatives that will most enhance the overall resilience of the road network. Table 4 lists the mean and percentage increase of the network WIPW for each combination of possible selections. While all possible solutions improve the overall network performance 
significantly, the combination of $\mathrm{S} 2+\mathrm{S} 4$ is most effective, with a $72.3 \%$ increase in WIPW from 0.61 to 1.05 .

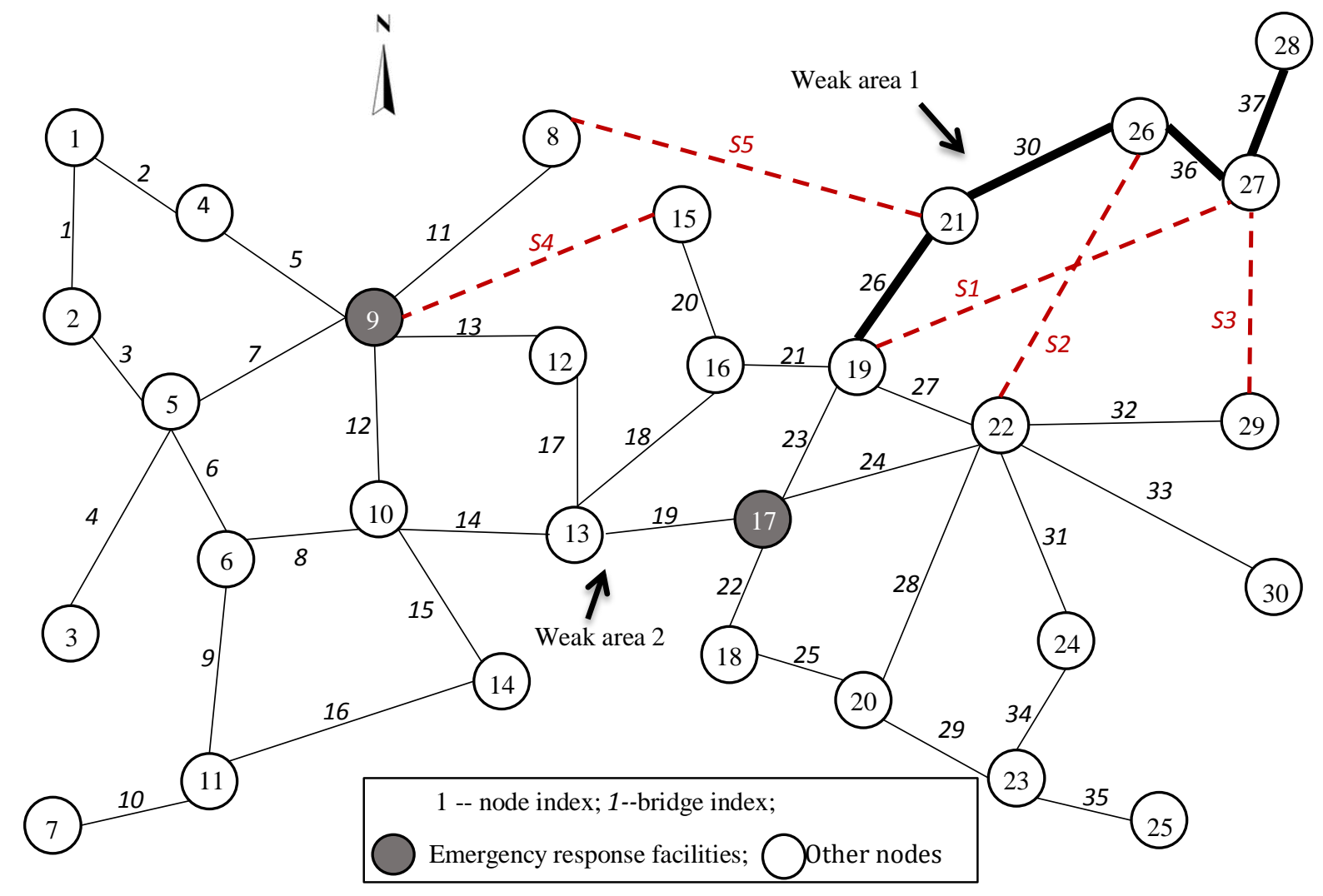

Figure 7. Hypothetical Bridge Network with Candidates for New Construction

Table 4. Impact of New Construction on Network Resilience

\begin{tabular}{|c|c|c|}
\hline $\begin{array}{c}\text { Possible } \\
\text { combinations }\end{array}$ & Mean WIPW & $\begin{array}{c}\text { Percentage of increase } \\
\text { in WIPW }\end{array}$ \\
\hline$S 1+S 4$ & 1.03 & $68.8 \%$ \\
\hline$S 1+S 5$ & 0.88 & $44.3 \%$ \\
\hline$S 2+S 4$ & 1.05 & $72.3 \%$ \\
\hline$S 2+S 5$ & 0.89 & $45.9 \%$ \\
\hline$S 3+S 4$ & 1.01 & $65.6 \%$ \\
\hline$S 3+S 5$ & 0.85 & $39.3 \%$ \\
\hline
\end{tabular}




\section{Conclusion}

This study introduced the WIPW, a quantitative resilience-based performance metric based on graph theory, to measure road network performance capacity following extreme natural hazard events. The WIPW systematically integrates the network topology, redundancy level, traffic patterns, location of community emergency response facilities, as well as failure probability of individual bridges. When coupled with risk mitigation decision analysis, risk mitigation alternatives for improving the resilience of a transportation system can be evaluated and compared on a common and rational basis. The decision framework in this study for effective risk mitigation through bridge retrofitting or new construction is formulated as a multiobjective optimization problem, which allows tradeoffs to be made between competing performance and cost objectives. The subjective preferences of the decision maker can be factored into the decision process through the selection of the weights on links and nodes. The formulation of the WIPW clearly suggest that the network resilience-based performance can be improved by: (1) increasing reliabilities of critical network components through appropriate retrofitting, (2) optimizing network topology through new construction, (3) altering traffic flow patterns through appropriate routing policies, and (4) strategically siting emergency response facilities [The (3) and (4) are not explicitly illustrated in the study, but are clearly reflected in the formulation of WIPW]. The WIPW effectively quantifies the performance capacity of a damaged network against the expected pre-disaster performance level (the "norm"). The weighting mechanism for nodes and links embedded in the formulation of WIPW provides a transparent framework to factor other network attributes (in addition to those discussed herein) that are important to specific communities into resiliencebased decision process, systematically and rationally.

\section{Acknowledgements}

The research reported herein was supported, in part, by the Center for Risk-Based Community Resilience Planning, funded by the National Institute of Standards and Technology (NIST). This support is gratefully acknowledged. The authors are grateful for conversations with Prof. Charles Nicholson, in the Department of Industrial and Systems Engineering at OU, which inspired the development of the optimization algorithm used in this study. However, the opinions expressed in this paper are solely those of the authors, and may not reflect the views of the sponsor.

\section{References}


Adachi, T. and Ellingwood B. R. (2008). "Serviceability of earthquake-damaged water systems: Effects of electrical power availability and power backup systems on system vulnerability." Reliability Engineering and System Safety 93(1):78-88.

Asakura, Y., and Kashiwadani, M. (1995). Traffic assignment in a road network with degraded links by natural disasters. Journal of the Eastern Asia Society for Transportation Studies, 1(3), 1135-1152.

Bocchini, P. and Frangopol, D. M. (2013) "Connectivity-Based Optimal Scheduling for Maintenance of Bridge Networks.” Journal of Engineering Mechanics, Vol. 139, No. 6, pp.760-769.

Bocchini, P. and Frangopol, D. M. (2011). "A stochastic computational framework for the joint transportation network fragility analysis and traffic flow distribution under extreme events." Probabilistic Engineering Mechanics, 26 (2011) 182-193

Bonstrom, H. and Corotis, R. B. (2014) Building portfolio seismic loss assessment using the First-Order Reliability Method. Structural Safety, Volume 52, Part A, January 2015, Pages 113-120.

Bruneau, M., S. Chang, R. Eguchi, G. Lee, T. O’Rourke, A.M. Reinhorn, M. Shinozuka, K. Tierney, W. Wallace, and D.V. Winterfelt. (2003). "A Framework to Quantitatively Assess and Enhance the Seismic Resilience of Communities.” Earthquake Spectra, 19 (4); 733-752.

Chang, S.E. and Nojima, N. (2001) "Measuring post-disaster transportation system performance: The 1995 Kobe earthquake in comparative perspective." Transportation Research Part A: Policy Pract, 35A $1-19$.

Chang, S. and Shinozuka, M. (2004). "Measuring improvements in the disaster resilience of communities." Earthquake Spectra 20(3):739-755.

Chen, A., Yang, H., Lo, H. K., and Tang, W. H. (2002). Capacity reliability of a road network: an assessment methodology and numerical results. Transportation Research Part B: Methodological, $36(3), 225-252$.

Chen, A., Kim, J., Zhou, Z., \& Chootinan, P. (2007). Alpha Reliable Network Design Problem. Transportation Research Record, 2029, 49-57.

Clark, S., and Watling, D. (2005). Modelling network travel time reliability under stochastic demand. Transportation Research Part B: Methodological, 39(2), 119-140.

Deb, K., Pratap, A., Agarwal, S., and Meyarivan, T. A. M. T. (2002). A fast and elitist multiobjective genetic algorithm: NSGA-II. Evolutionary Computation, IEEE Transactions on, 6(2), 182-197.

DHS (2009) HAZUS-MH MR4 Emergency Management earthquake model user manual. Washington (DC): Department of Homeland Security, Federal Agency, Mitigation Division.

Eiben, A. E., and Smit, S. K. (2012). Evolutionary algorithm parameters and methods to tune them. In Autonomous Search (pp. 15-36). Springer Berlin Heidelberg.

Fragkakis, N. P., and Lambropoulos, S. (2004). "A quantity and cost estimate model for concrete road bridges.” Technica Chronica I: Scientific J. Technical Chamber, Greece, 2-3, 65-77. 
Ghosh, J., Rokneddin, K., Padgett, J. E., \& Dueñas-Osorio, L. (2014). Seismic Reliability Assessment of Aging Highway Bridge Networks with Field Instrumentation Data and Correlated Failures, I: Methodology. Earthquake Spectra, 30(2), 795-817.

Gibbons AM. Algorithmic graph theory. Cambridge University Press; 1985.

Guikema, S., and Gardoni, P. (2009). Reliability estimation for networks of reinforced concrete bridges. Journal of Infrastructure Systems, 15(2), 61-69.

Holling CS. (1973) "Resilience and stability of ecological systems." Annual Review of Ecology and Systematics 1973; 4(1):1-23.

Ip, W. H., and Wang, Q. (2011). Resilience and friability of transportation networks: evaluation, analysis and optimization. Systems Journal, IEEE, 5(2), 189-198.

Jayaram, N. and J.W. Baker. (2009). "Correlation Model for Spatially Distributed Ground-motion Intensities." Earthquake Engineering \& Structural Dynamics, 38(15); 22.

Koski, J., R. Silvennoinen. 1987. Norm methods and partial weighting in multicriterion optimization of structures. International Journal for Numerical Methods in Engineering 24(6) 1101-1121.

Kurtz, N., Song, J., \& Gardoni, P. (2015). Seismic Reliability Analysis of Deteriorating Representative US West Coast Bridge Transportation Networks.Journal of Structural Engineering, C4015010.

Lee, R. and Kiremidjian, A.S. (2007). , "Uncertainty and Correlation for Loss Assessment of Spatially Distributed Systems," Earthquake Spectra, vol. 23, pp. 753-770.

Lee, Y. J., Song, J., Gardoni, P., \& Lim, H. W. (2011). Post-hazard flow capacity of bridge transportation network considering structural deterioration of bridges. Structure and Infrastructure Engineering, 7(7-8), 509-521.

Leichenko, R. (2011). Climate change and urban resilience. Current Opinion in Environmental Sustainability, 3(3), 164-168.

MacGregor, J.G., S.A. Mirza and B. R. Ellingwood (1983), "Statistical analysis of resistance of reinforced and prestressed concrete members," J. American Concrete Inst. 80(3):167-176.

Marler, T., J. Arora. 2010. The weighted sum method for multi-objective optimization: new insights. Structural and multidisciplinary optimization 41(6) 853-862.

Martin, R. (2012). Regional economic resilience, hysteresis and recessionary shocks. Journal of Economic Geography, 12(1), 1-32.

Mesbah, M., Sarvi, M., \& Currie, G. (2011). Optimization of transit priority in the transportation network using a genetic algorithm. Intelligent Transportation Systems, IEEE Transactions on, 12(3), 908919.

Miller, A. and Xiao, Y. (2007) "Multi-level strategies to achieve resilience for an organization operating at capacity: A case study at a trauma centre," Cogn. Tech. Work., vol. 9, pp. 51-66. 
Morlok, EK and Chang, DJ (2004) "Measuring capacity flexibility of a transportation system," Transport. Res. A, vol. 38, pp. 405-420.

Nagurney, A., and Qiang, Q. (2007). A network efficiency measure for congested networks. EPL (Europhysics Letters), 79(3), 38005.

Padgett, J. E., and DesRoches, R. (2009). Retrofitted bridge fragility analysis for typical classes of multispan bridges. Earthquake Spectra, 25(1), 117-141.

Peeta, S., Sibel, S. F., Gunnec, D., and Viswanath, K. (2010) "Pre-disaster Investment Decisions for Strengthening a Highway Network" Computers \&Operations Research, 37(2010) 1708-1719.

Pishvaee, M. S., Farahani, R. Z., \& Dullaert, W. (2010). A memetic algorithm for bi-objective integrated forward/reverse logistics network design.Computers \& operations research, 37(6), 1100-1112.

Pimm, S. (1984). “The Complexity and Stability of Ecosystems.” Nature, 307(5949); 321-326.

Osman, I. H., \& Kelly, J. P. (Eds.). (2012). Meta-heuristics: theory and applications. Springer Science \& Business Media.

Quanyan Zhu and Tamer Basar (2015) "Game-Theoretic Methods for Robustness, Security, and Resilience of Cyberphysical Control Systems" IEEE Control System Magazine, DOI 10.1109/MCS.2014.2364710

Reggiani, A. (2002) "Resilience: An evolutionary approach to spatial economic systems," Netw. Spatial Econ., vol. 2, pp. 211-229.

Rokneddin, K., Ghosh, J., Dueñas-Osorio, L., \& Padgett, J. E. (2014). Seismic Reliability Assessment of Aging Highway Bridge Networks with Field Instrumentation Data and Correlated Failures, II: Application. Earthquake Spectra, 30(2), 819-843.

Rose A. (2007) "Economic resilience to natural and man-made disasters: multi-disciplinary origins and contextual dimensions." Environmental Hazards 2007; 7(4):383-98.

Shinozuka, M., Feng, M. Q., Lee, J., and Naganuma, T. (2000). Statistical analysis of fragility curves. Journal of Engineering Mechanics, 126(12), 1224-1231.

Shiraki, N., Shinozuka, M., Moore, J.E., Chang, S.E., Kameda, H., and Tanaka, S. (2007) "System Risk Curves: Probabilistic Performance Scenarios for Highway Networks Subjected to Earthquake Damage" Journal of Infrastructure System, Vol. 13 No. 1 pp 43-54.

Simonis, H., "Constraint based resilience analysis," Lecture Notes in Computer Science, vol. 4204, pp. 16$28,2006$.

Skiena, S. (1990). Dijkstra's Algorithm. Implementing Discrete Mathematics: Combinatorics and Graph Theory with Mathematica, Reading, MA: Addison-Wesley, 225-227. 
Sohn J., Kim, T.J., Hewings, G. J. D., Lee, J. S. and Jang, S. (2003) "Retrofit Priority of Transport Network Links under an Earthquake" Journal of Urban Planning and Development, Vol. 129, No. 4, pp 195210.

Sterbenz, J. P., Hutchison, D., Çetinkaya, E. K., Jabbar, A., Rohrer, J. P., Schöller, M., \& Smith, P. (2010). Resilience and survivability in communication networks: Strategies, principles, and survey of disciplines. Computer Networks,54(8), 1245-1265.

Timmerman, P. (1981). "Vulnerability. Resilience and the collapse of society: A review of models and possible climatic applications." Environmental Monograph, Institute for Environmental Studies, Univ. of Toronto, Canada.

Vitoontus, S. and Ellingwood B.R. (2013). "Role of Correlation in Seismic Demand and Building Damage in Estimating Losses under Scenario Earthquakes." Proc. Int. Conf. on Struct. Safety and Reliability (ICOSSAR 2013), New York, NY, Taylor \& Francis, A.A. Balkema, The Netherlands.

Walker, B. and Salt, D. (2006). Resilience thinking: sustaining ecosystems and people in a changing world. Island Press.

Zadeh, L. 1963. Optimality and non-scalar-valued performance criteria. IEEE Transactions on Automatic Control 8(1) 59-60.

Zhang, W and Wang, N (2015) "Bridge Network Maintenance Optimization under Budget Constraint," ASCE Journal of Infrastructure Systems, in review, 2015. 